\title{
Two Kinds of New Integrable Couplings of the Negative-Order Korteweg-de Vries Equation
}

\author{
Binlu Feng ${ }^{1}$ and Yufeng Zhang ${ }^{2}$ \\ ${ }^{1}$ School of Mathematics and Information Sciences, Weifang University, Weifang 261061, China \\ ${ }^{2}$ College of Sciences, China University of Mining and Technology, Xuzhou 221116, China \\ Correspondence should be addressed to Binlu Feng; fbl_1963@126.com
}

Received 28 October 2014; Revised 25 November 2014; Accepted 17 December 2014

Academic Editor: Stephen C. Anco

Copyright (c) 2015 B. Feng and Y. Zhang. This is an open access article distributed under the Creative Commons Attribution License, which permits unrestricted use, distribution, and reproduction in any medium, provided the original work is properly cited.

Based on some known loop algebras with finite dimensions, two different negative-order integrable couplings of the negativeorder Korteweg-de Vries (KdV) hierarchy of evolution equations are generated by making use of the Tu scheme, from which the corresponding negative-order integrable couplings of the negative-order KdV equations are followed to be obtained. The resulting Hamiltonian structure of one negative integrable coupling is derived from the variational identity.

\section{Introduction}

Since the concept on integrable couplings was proposed [1], some integrable couplings of the known integrable systems, such as the AKNS system and the KN system, were obtained. Ma [2] made use of the perturbation method to obtain the integrable couplings of the $\mathrm{KdV}$ equation as well as their some algebraic and geometric properties. Reference [3] employed a simple finite-dimensional Lie algebra to present a method for generating integrable couplings of integrable hierarchies of evolution equations. Later, Guo and Zhang [4] proposed the quadratic-form identity for which Hamiltonian structures of some integrable couplings were generated. Ma and Chen [5] further generalized the quadratic-form identity and completely improved it to obtain the variational identity for deducing the Hamiltonian structures of integrable couplings which is more convenient. Actually, there are lots of related references in integrable-coupling area. The adopted algebras were studied and applied in other existing literature. One of them is so-called perturbation algebra, which was even used to present $2+1$-dimensional local biHamiltonian systems by Ma and Fuchssteiner in 1996, and whose systematical presentation was presented in [6]. In addition, $\mathrm{Ma}$ et al. [2, 7-9] introduced different Lie algebras to obtain some new and interesting integrable couplings. By following the methods, many interesting integrable couplings of some known integrable hierarchies were obtained, such as the works in $[10-12]$. However, to our best knowledge, the negative-order integrable couplings of the negative-order integrable hierarchies have not been discussed systematically. Up to now, we know that some interesting related negativeorder integrable equations including the negative-order $\mathrm{KdV}$ equation and some associated properties were obtained, such as the results in [13-18]. But their negative-order integrable couplings have not been discussed. In the paper, we want to discuss the problem. Qiao and Fan [18] employed the Lenard sequence method to obtain the negative-order $\mathrm{KdV}$ equation and then represented it by its Lax pair. Enlightened by this work, we will generate the negative-order KdV hierarchy and its integrable couplings by enlarged Lie algebras and the enlarged Lax pairs. It is remarkable that the integrable couplings obtained in the paper include the linear and nonlinear integrable couplings. In the aspect of nonlinear integrable couplings, Ma and Zhu [19] proposed some semisimple Lie algebras to present a way for generating nonlinear discrete integrable couplings. Then [20-22] also reveal some nonlinear integrable couplings by constructing various Lie algebras. As reduced cases, two negative-order integrable couplings of the negative-order KdV equation are obtained. Finally, the Hamiltonian structure of one negative-order integrable coupling in the negative-order KdV hierarchy is obtained by the variational identity. 


\section{Two Different Negative-Order Integrable Couplings}

In the section, we adopt the Tu scheme [23] which was proposed by Ma [24] and the known finite-dimensional Lie algebras to deduce two integrable couplings of the negativeorder KdV hierarchy. As reduced cases, two negative-order integrable couplings of the negative-order $\mathrm{KdV}$ equation are also followed to present. In $[25,26]$, a 6-dimensional Lie algebra reads

$$
G_{1}=\left\{g_{1}, g_{2}, \ldots, g_{6}\right\}
$$

where

$$
\begin{aligned}
& g_{1}=\left(\begin{array}{cc}
h_{1} & 0 \\
0 & h_{2}
\end{array}\right), \\
& g_{2}=\left(\begin{array}{cc}
h_{2} & 0 \\
0 & h_{2}
\end{array}\right), \\
& g_{3}=\left(\begin{array}{cc}
h_{3} & 0 \\
0 & h_{3}
\end{array}\right), \\
& g_{4}=\left(\begin{array}{cc}
0 & h_{1} \\
0 & 0
\end{array}\right), \\
& g_{5}=\left(\begin{array}{cc}
0 & h_{2} \\
0 & 0
\end{array}\right), \\
& g_{6}=\left(\begin{array}{cc}
0 & h_{3} \\
0 & 0
\end{array}\right) ;
\end{aligned}
$$

here $h_{1}=\left(\begin{array}{cc}1 & 0 \\ 0 & -1\end{array}\right), h_{2}=\left(\begin{array}{ll}0 & 1 \\ 0 & 0\end{array}\right)$, and $h_{3}=\left(\begin{array}{ll}0 & 0 \\ 1 & 0\end{array}\right)$.

In [26], another 6-dimensional Lie algebra was given by

$$
G_{2}=\left\{f_{1}, f_{2}, \ldots, f_{6}\right\}
$$

where

$$
\begin{aligned}
& f_{i}=g_{i}, \quad i=1,2,3 ; \\
& f_{4}=\left(\begin{array}{ll}
0 & h_{1} \\
0 & h_{1}
\end{array}\right), \\
& f_{5}=\left(\begin{array}{ll}
0 & h_{2} \\
0 & h_{2}
\end{array}\right), \\
& f_{6}=\left(\begin{array}{ll}
0 & h_{3} \\
0 & h_{3}
\end{array}\right) .
\end{aligned}
$$

The resulting loop algebras can be defined as $p_{i}(n)=p_{i} \otimes \lambda^{n}$, where $p_{i} \in G_{1}$ or $p_{i} \in G_{2}$. The loop algebras of the Lie algebras $G_{1}$ and $G_{2}$ are denoted by $\widetilde{G}_{1}$ and $\widetilde{G}_{2}$, respectively. According to the Tu scheme, we first solve a stationary 0curvature equation

$$
V_{x}=[U, V] \text {, }
$$

where the Lax matrices $U$ and $V$ belong to the loop algebra $\widetilde{G}_{1}$ or $\widetilde{G}_{2} ; U$ and $V$ can be represented by the loop algebra $\widetilde{G}_{1}$ or $\widetilde{G}_{2}$ :

$$
\begin{aligned}
& U=R+\sum_{i=1}^{6} u_{i} p_{i}(n), \\
& V=\sum_{m=0}^{\infty}\left(\sum_{i=1}^{6} V_{i m} p_{i}(-m)\right),
\end{aligned}
$$

where $R$ and $p_{i}(n)$ belong to $\widetilde{G}_{1}$ or $\widetilde{G}_{2}$ and require $\operatorname{deg}(R) \geq$ $\operatorname{deg}\left(p_{i}(n)\right)$; here deg stands for the degree of the elements in the loop algebras; $V_{i m}$ are the derivative functions in $x$ and $t$. Then choose a suitable modified term $\Delta_{n}$ so that

$$
V^{(n)}=V_{+}^{(n)}+\Delta_{n}
$$

where

$$
V_{+}^{(n)}=\sum_{m=0}^{n}\left(\sum_{i=1}^{6} V_{i m} p_{i}(n-m)\right)=\lambda^{n} V-V_{-}^{(n)}
$$

satisfies the 0 -curvature equation

$$
U_{t}-V_{x}^{(n)}+\left[U, V^{(n)}\right]=0
$$

which can derive an integrable hierarchy of evolution equations. In order to deduce negative-order integrable hierarchies, we need to change (7) to the following form:

$$
V=\sum_{m=-\infty}^{0} V_{m} \lambda^{-m}=: \sum_{m \leq 0} V_{i m} p_{i}(-m) .
$$

Qiao and $\mathrm{Li}$ [17] once obtained the negative-order $\mathrm{KdV}$ equation through the Lenard recursive sequence

$$
\begin{aligned}
& v_{t}=w_{x} \\
& w_{x x x}+4 v w_{x}+2 v_{x} w=0
\end{aligned}
$$

which is equivalent to a nonlinear quartic integrable system

$$
u u_{x x t}-u_{x x} u_{t}-2 u^{3} u_{x}=0 .
$$

The Lax pair of (12) was presented by a proposition in [18]. Furthermore, Qiao and Fan produced the Hamiltonian structure, Darboux transformations, and some exact solutions. Enlightened by their results, we will deduce the negativeorder KdV hierarchy by employing the Tu scheme which can reduce to the negative-order $\mathrm{KdV}$ equation (12).

Denote $\widetilde{H}=\operatorname{span}\left\{h_{1}(n), h_{2}(n), h_{3}(n),\right\}$ where $h_{i}(n)=$ $h_{i} \lambda^{n}, i=1,2,3$; then $\widetilde{H}$ is an obvious loop algebra. Employing the loop algebra, we introduce a pair of Lax matrices

$$
\begin{aligned}
& U=h_{3}(1)-u h_{3}(0)+h_{2}(0), \\
& V=\sum_{m=-\infty}^{0}\left(a_{m} h_{1}(-m)+b_{m} h_{2}(-m)+c_{m} h_{3}(-m)\right) .
\end{aligned}
$$


A solution to (5) is presented as

$$
\begin{aligned}
b_{m+1} & =-a_{m, x}+u b_{m}+c_{m}, \\
b_{m, x} & =-2 a_{m}, \\
2 a_{m+1} & =c_{m, x}+2 u a_{m}, \quad m=0,-1,-2, \ldots,
\end{aligned}
$$

which is equivalent to the following relation:

$$
J b_{m+1}=K b_{m}
$$

where

$$
\begin{aligned}
J & =\partial=\frac{\partial}{\partial x} \\
K & =\frac{1}{4} \partial^{3}+\frac{1}{2}(u \partial+\partial u)
\end{aligned}
$$

Equation (16) is called the Lenard sequence [18]. Denote

$$
\begin{array}{r}
V^{(-n)}=\lambda^{-n} V-V_{-}^{(-n)}=\sum_{m=-n+1}^{0} V_{m} \lambda^{-n-m}, \\
n=1,2,3, \ldots .
\end{array}
$$

Equation (5) can be decomposed into

$$
-\left(V_{+}^{(-n)}\right)_{x}+\left[U, V_{+}^{(-n)}\right]=\left(V_{-}^{(-n)}\right)_{x}-\left[U, V_{-}^{(-n)}\right] .
$$

With the help of (14) and (19), we get that

$$
-\left(V_{+}^{(-n)}\right)_{x}+\left[U, V_{+}^{(-n)}\right]=-2 a_{1-n} h_{3}(0)+b_{1-n} h_{1}(0) .
$$

Take

$$
\begin{aligned}
\Delta_{n} & =-b_{1-n} h_{1}(0), \\
V^{(-n)} & =V_{+}^{(-n)}+\Delta_{n} ;
\end{aligned}
$$

we have that

$$
\begin{aligned}
-V_{x}^{(-n)}+\left[U, V^{(-n)}\right] & =\left[\left(b_{1-n}\right)_{x}-2 a_{1-n}\right] h_{3}(0) \\
& =2\left(b_{1-n}\right)_{x} h_{3}(0) .
\end{aligned}
$$

Therefore, the 0 -curvature equation

$$
U_{t}-V_{x}^{(-n)}+\left[U, V^{(-n)}\right]=0
$$

admits the following integrable hierarchy:

$$
u_{t_{n-1}}=2\left(b_{1-n}\right)_{x}, \quad n=1,2,3, \ldots,
$$

which is called the negative-order KdV hierarchy. Obviously, we have

$$
\begin{aligned}
& u_{t_{0}}=2 b_{0, x}, \\
& u_{t_{1}}=2 b_{-1, x}, \\
& u_{t_{2}}=2 b_{-2, x},
\end{aligned}
$$

When $m=0$, (16) gives $b_{0}=0, b_{1, x}=0$; here let $b_{1}=0$.
When $m=-1$, (16) reduces to

$$
b_{0, x}=\frac{1}{4} b_{-1, x x x}+\frac{1}{2} u b_{-1, x}+\frac{1}{2}\left(u b_{-1}\right)_{x} .
$$

Assume $b_{-1}=w$; then (26) becomes

$$
w_{x x x}+4 u w_{x}+2 u_{x} w=0 .
$$

Combined with (25), we obtain the negative-order $\mathrm{KdV}$ equation when $t_{1}=t$ :

$$
\begin{aligned}
& u_{t}=2 w_{x}, \\
& w_{x x x}+4 u w_{x}+2 u_{x} w=0,
\end{aligned}
$$

which is the same as (12) except for a constant 2 in the first equation. Qiao and Fan [18] established a Lax pair of the negative-order $\mathrm{KdV}$ equation by employing the Lenard sequence method.

In what follows, we can directly single out the Lax pair of (28). It is easy to see that

$$
\begin{aligned}
V^{(-2)}= & \sum_{m=-1}^{0}\left(a_{m} h_{1}(-2-m)+b_{m} h_{2}(-2-m)\right. \\
& \left.+c_{m} h_{3}(-2-m)\right)-b_{-1} h_{3}(0) \\
= & a_{0} h_{1}(-2)+b_{0} h_{2}(-2)+c_{0} h_{3}(-2) \\
& +a_{-1} h_{1}(-1)+b_{-1} h_{2}(-1)+c_{-1} h_{3}(-1)-b_{-1} h_{3}(0) \\
= & \left(\begin{array}{cc}
q_{-1} \lambda^{-1} & b_{-1} \lambda^{-1} \\
c_{-1} \lambda^{-1}-b_{-1} & -a_{-1} \lambda^{-1}
\end{array}\right) .
\end{aligned}
$$

Due to $b_{0}=0$, we can get $a_{0}=0$. In terms of (15), we have $a_{-1}=-(1 / 2) w_{x}, c_{-1}=-(1 / 2) w_{x x}-u w$. Thus, one infers that

$$
V^{(-2)}=\left(\begin{array}{cc}
-\frac{1}{2} w_{x} \lambda^{-1} & w \lambda^{-1} \\
-\left(\frac{1}{2} w_{x x}+u w\right) \lambda^{-1}+w & \frac{1}{2} w_{x} \lambda^{-1}
\end{array}\right) .
$$

Hence, the compatibility condition of the Lax pair

$$
\begin{aligned}
\varphi_{x} & =U \varphi, \quad U=\left(\begin{array}{cc}
0 & 1 \\
\lambda-u & 0
\end{array}\right), \\
\varphi_{t} & =V^{(-2)} \varphi,
\end{aligned}
$$

gives rise to the negative-order $\mathrm{KdV}$ equation (28); that is, the 0 -curvature equation

$$
U_{t}-V_{x}^{(-2)}+\left[U, V^{(-2)}\right]=0
$$

admits (28). The Hamiltonian structure of (28) can be given similar to that in [18]; here we do not repeat it again.

\section{Two Negative-Order Integrable Couplings}

We first make use of the loop algebra $\widetilde{G}_{1}$ to deduce the first negative-order integrable couplings of the negative-order $\mathrm{KdV}$ hierarchy and then employ the second loop algebra $\widetilde{G}_{2}$ to derive the second negative-order integrable couplings. 
3.1. The First Negative-Order Integrable Coupling. Set

$$
\begin{aligned}
& U=g_{3}(1)-u g_{3}(0)+g_{2}(0)+r g_{6}(0), \\
& V=\sum_{m=-\infty}^{0}\left(\sum_{i=1}^{6} V_{i m} g_{i}(-m)\right) ;
\end{aligned}
$$

then (5) gives the following recursive relations among $V_{\text {im }}(i=1,2, \ldots, 6)$ :

$$
\begin{aligned}
& \left(V_{1 m}\right)_{x}=-V_{2, m+1}+u v_{2 m}+V_{3 m} \\
& \left(V_{2 m}\right)_{x}=-2 V_{1 m} \\
& \left(V_{3 m}\right)_{x}=2 V_{1, m+1}-2 u V_{1 m}, \quad m=0,-1,-2, \ldots ; \\
& \left(V_{4 m}\right)_{x}=V_{5, m+1}+u V_{5 m}+V_{6 m}-r V_{2 m}, \\
& \left(V_{5 m}\right)_{x}=-2 V_{4 m}, \\
& \left(V_{6 m}\right)_{x}=2 V_{4, m+1}-2 u V_{4 m}+2 r V_{1 m}, \quad m=0,-1,-2, \ldots ;
\end{aligned}
$$

here (34) is just the same as (15). Denote

$$
\begin{aligned}
V^{(-n)}= & \sum_{m=-n+1}^{0}\left(\sum_{i=1}^{6} V_{i m} g_{i}(-n-m)\right)-V_{2,1-n} g_{3}(0) \\
& -V_{5,1-n} g_{4}(0) ;
\end{aligned}
$$

after tedious calculation, one infers that

$$
\begin{aligned}
-V_{x}^{(-n)}+\left[U, V^{(-n)}\right]= & {\left[\left(V_{2,1-n}\right)_{x}-2 V_{1,1-n}\right] g_{3}(0) } \\
& +\left[\left(V_{5,1-n}\right)_{x}-2 V_{4,1-n}\right] g_{6}(0)
\end{aligned}
$$

Therefore, the 0 -curvature equation (10) is equivalent to

$$
\begin{aligned}
& u_{t_{n-1}}=\left(V_{2,1-n}\right)_{x}-2 V_{1,1-n}=2\left(V_{2,1-n}\right)_{x}, \\
& r_{t_{n-1}}=-\left(V_{5,1-n}\right)_{x}+2 V_{4,1-n}=-2\left(V_{5,1-n}\right)_{x},
\end{aligned}
$$

which is the negative-order integrable couplings of the negative-order KdV hierarchy. In what follows, we want to consider its reduction. First of all, we have a recursive relation from (35):

$$
\begin{aligned}
\left(V_{5, m+1}\right)_{x}= & \frac{1}{4}\left(V_{5 m}\right)_{x x x} \\
& +\frac{1}{2}\left[u\left(V_{5 m}\right)_{x}+\left(u V_{5 m}\right)_{x}-r\left(V_{2 m}\right)_{x}-\left(r V_{2 m}\right)_{x}\right] .
\end{aligned}
$$

When $m=0$, we take $V_{2,0}=V_{2,1}=V_{5,0}=V_{5,1}=0$. When $m=-1$, we have in terms of (39) that

$$
\begin{aligned}
&\left(V_{5,0}\right)_{x}= \frac{1}{4}\left(V_{5,-1}\right)_{x x x} \\
&+\frac{1}{2}\left[u\left(V_{5,-1}\right)_{x}+\left(u V_{5,-1}\right)_{x}\right. \\
&\left.\quad-r\left(V_{2,-1}\right)_{x}-\left(r V_{2,-1}\right)_{x}\right] .
\end{aligned}
$$

Let $V_{5,-1}=-q$; we find from (40) that

$$
q_{x x x}+2 u q_{x}+2(q u)_{x}+2 r w_{x}+2(r w)_{x}=0 .
$$

Assume $t_{1}=t$; since $r_{t}=-2 q_{x}$, thus we have

$$
\begin{array}{r}
w_{x x x}+4 u w_{x}+2 u_{x} w, \quad u_{t}=2 w_{x}, \\
q_{x x x}+2 u q_{x}+2(q u)_{x}+2 r w_{x}+2(r w)_{x}=0, \\
r_{t}=-2 q_{x} .
\end{array}
$$

The first equation in (42) is the negative-order KdV equation, while the second equation is just coupled part of integrable couplings. Therefore, (42) is an integrable coupling of the negative-order $\mathrm{KdV}$ equation.

\subsection{The Second Negative-Order Integrable Coupling. Set}

$$
\begin{aligned}
& U=f_{3}(1)-u f_{3}(0)+f_{2}(0)+v f_{6}(0), \\
& V=\sum_{m \leq 0}\left(\sum_{i=1}^{6} V_{i m} f_{i}(-m)\right) .
\end{aligned}
$$

Similar to the previous case, we can get (34) and the following equations which are different from (35):

$$
\begin{aligned}
& \left(V_{4 m}\right)_{x}=-V_{5, m+1}+(u-v) V_{5 m}+V_{6 m}-v V_{2 m}, \\
& \left(V_{5 m}\right)_{x}=-2 V_{4 m}, \\
& \left(V_{6 m}\right)_{x}=2 V_{4, m+1}+(2 v-2 u) V_{4 m}+2 v V_{1 m}, \\
& m=0,-1,-2, \ldots,
\end{aligned}
$$

from which we have

$$
\begin{aligned}
\left(V_{5, m+1}\right)_{x}= & \frac{1}{4}\left(V_{5 m}\right)_{x x x} \\
& +\frac{1}{2}\left[(u-v)\left(V_{5 m}\right)_{x}+\left((u-v) V_{5 m}\right)_{x}\right. \\
& \left.\quad-\left(V_{2 m}\right)_{x}-\left(v V_{2 m}\right)_{x}\right] .
\end{aligned}
$$

Take $V_{5,0}=V_{2,0}=V_{2,1}=0$; we have

$$
\begin{aligned}
\left(V_{5,0}\right)_{x}= & \frac{1}{4}\left(V_{5,-1}\right)_{x x x} \\
+ & \frac{1}{2}\left[(u-v)(V-5,-1)_{x}+\left((u-v) V_{5,-1}\right)_{x}\right. \\
& \left.\quad-\left(V_{2,-1}\right)_{x}-\left(v V_{2,-1}\right)_{x}\right] .
\end{aligned}
$$

Set $V_{5,-1}=-s$; one gets from (46) that

$$
s_{x x x}+2(u-v) s_{x}+(u s-v s)_{x}+w_{x}+(v w)_{x}=0 .
$$

Denote

$$
\begin{aligned}
V^{(-n)}= & \sum_{m=-n+1}^{0}\left(\sum_{i=1}^{6} V_{i m} f_{i}(-n-m)\right)-V_{2,1-n} f_{3}(0) \\
& -V_{5,1-n} f_{6}(0)
\end{aligned}
$$


one infers that

$$
\begin{aligned}
-V_{x}^{(-n)}+\left[U, V^{(-n)}\right]= & {\left[\left(V_{2,1-n}\right)_{x}-2 V_{1,1-n}\right] f_{3}(0) } \\
& +\left[\left(V_{5,1-n}\right)_{x}-2 V_{4,1-n}\right] f_{6}(0)
\end{aligned}
$$

Thus, the 0 -curvature equation

$$
U_{t}-V_{x}^{(-n)}+\left[U, V^{(-n)}\right]=0
$$

gives that

$$
\begin{aligned}
& u_{t_{n-1}}=2\left(V_{2,1-n}\right)_{x}, \\
& v_{t_{n-1}}=-2\left(V_{5,1-n}\right)_{x}, \quad n=1,2, \ldots
\end{aligned}
$$

We call (51) a negative-order integrable coupling of the negative-order KdV hierarchy. A reduced case of (51) presents that when $n=1,2$,

$$
\begin{gathered}
w_{x x x}+4 u w_{x}+2 u_{x} w=0, \quad u_{t}=2 w_{x}, \\
s_{x x x}+2(u-v) s_{x}+(u s-v s)_{x}+w_{x}+(v w)_{x}=0, \\
v_{t}=-2 s_{x},
\end{gathered}
$$

which is the second negative-order integrable coupling of the negative-order KdV equation. As comparison, we rewrite the second equation in (42) as follows:

$$
r_{x x t}+4 u r_{t}-8 w_{x} r-4 w r_{x}+2 u_{x} \int^{x} r_{t} d x=0
$$

which is linear with respect to the dependent variable $r$. The second coupled part in (52) presents again

$$
\begin{aligned}
v_{x x t}+ & 3(u-v) v_{t}-2 w_{x}-2 w v_{x}-2 w_{x} v \\
& +(u-v)_{x} \int^{x} v_{t} d x=0,
\end{aligned}
$$

which is nonlinear in the variable $v$. Therfore, we obtain the linear integrable coupling of the negative-order $\mathrm{KdV}$ equation (28):

$$
\begin{aligned}
& u_{t}=2 w_{x}, \quad w_{x x x}+4 u w_{x}+2 u_{x} w=0, \\
& r_{x x t}+4 u r_{t}-8 w_{x} r-4 w r_{x}+2 u_{x} \int^{x} r_{t} d x=0,
\end{aligned}
$$

and the nonlinear integrable coupling:

$$
\begin{aligned}
& u_{t}=2 w_{x}, \quad w_{x x x}+4 u w_{x}+2 u_{x} w=0, \\
& v_{x x t}+3(u-v) v_{t}-2 w_{x}-2 w v_{x}-2 w_{x} v \\
& +(u-v)_{x} \int^{x} v_{t} d x=0 .
\end{aligned}
$$

Remark 1. As previous statement, Qiao and Fan [18] have obtained the Hamiltonian structure of the negative-order $\mathrm{KdV}$ equation by using the trace identity proposed by $\mathrm{Tu}$ [23]. In what follows, we will deduce the Hamiltonian structure of the whole negative-order integrable coupling (51) by employing the variational identity.

3.3. The Hamiltonian Structure of the Nonlinear Integrable Couplings. Rewrite (43) as the forms of square matrices

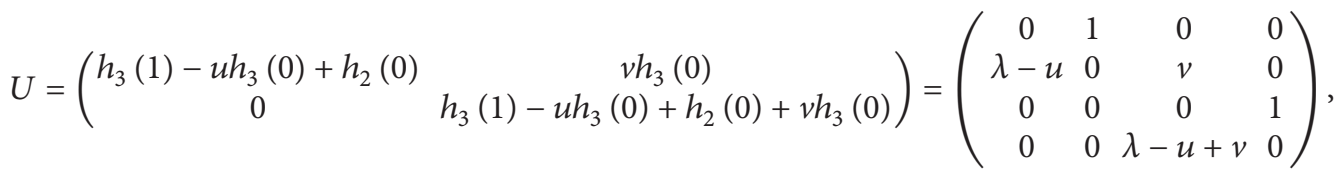

$$
\begin{aligned}
& V=\left(\begin{array}{cc}
\sum_{i=1}^{3} V_{i} h_{i} & \sum_{i=4}^{6} V_{i} h_{i-3} \\
0 & \sum_{i=1}^{3} V_{i} h_{i}+\sum_{i=4}^{6} V_{i} h_{i-3}
\end{array}\right)=\left(\begin{array}{cccc}
V_{1} & V_{2} & V_{4} & V_{5} \\
V_{3} & -V_{1} & V_{6} & -V_{4} \\
0 & 0 & V_{1}+V_{4} & V_{2}+V_{5} \\
0 & 0 & V_{3}+V_{6} & -V_{1}-V_{4}
\end{array}\right),
\end{aligned}
$$

where $V_{i}=\sum_{m=-\infty}^{-1} V_{i m} \lambda^{-m}, i=1,2, \ldots, 6$.

In order to employ the variational identity, we should establish a Lie algebra with collumn-vector elements. For that sake, we consider the linear space $R^{6}$ equipped with a kind of commutator as follows:

$$
[a, b]=\left(\begin{array}{l}
{[a, b]_{1}} \\
{[a, b]_{2}}
\end{array}\right)
$$

where

$$
\begin{aligned}
& a=\sum_{i=1}^{6} a_{i} f_{i}, \\
& b=\sum_{i=1}^{6} b_{i} f_{i} \in \widetilde{G}_{2},
\end{aligned}
$$

$$
\begin{aligned}
{[a, b]_{1}=} & \left(a_{2} b_{3}-a_{3} b_{2}, 2 a_{1} b_{2}-2 a_{2} b_{1}, 2 a_{3} b_{1}-2 a_{1} b_{3}\right)^{T}, \\
{[a, b]_{2}=} & \left(a_{2} b_{6}-a_{6} b_{2}+a_{5} b_{3}-a_{3} b_{5}+a_{5} b_{6}-a_{6} b_{5},\right. \\
& 2 a_{1} b_{5}-2 a_{5} b_{1}+2 a_{4} b_{2}-2 a_{2} b_{4}+2 a_{4} b_{5}-2 a_{5} b_{4}, \\
& \left.2 a_{3} b_{4}-2 a_{4} b_{3}+2 a_{6} b_{1}-2 a_{1} b_{6}+2 a_{6} b_{4}-2 a_{4} b_{6}\right)^{T} .
\end{aligned}
$$

The commutator (59) can be written as

$$
[a, b]=a^{T} R(b),
$$


where

$$
\begin{aligned}
a^{T} & =\left(a_{1}, a_{2}, \ldots, a_{6}\right), \\
R(b) & =\left(\begin{array}{ccc}
R_{11}(b) & R_{12}(b) \\
0 & R_{22}(b)
\end{array}\right), \\
R_{11}(b) & =\left(\begin{array}{ccc}
0 & 2 b_{2} & -2 b_{3} \\
b_{3} & -2 b_{1} & 0 \\
-b_{2} & 0 & 2 b_{1}
\end{array}\right), \\
R_{12}(b) & =\left(\begin{array}{ccc}
0 & 2 b_{5} & -2 b_{6} \\
b_{6} & -2 b_{4} & 0 \\
b_{5} & 0 & 2 b_{4}
\end{array}\right), \\
R_{22}(b) & =\left(\begin{array}{ccc}
0 & 2 b_{2}+2 b_{5} & -2 b_{3}-2 b_{6} \\
b_{3}+b_{6} & -2 b_{1}-2 b_{4} & 0 \\
-b_{2}-b_{5} & 0 & 2 b_{1}+2 b_{4}
\end{array}\right) .
\end{aligned}
$$

A direct verification indicates that the linear space $R^{6}$ becomes a Lie algebra with the commutator (59). It is obvious that the linear map

$$
\begin{aligned}
\delta: G_{2} & \longrightarrow R^{6}, \\
A & \longrightarrow\left(a_{1}, a_{2}, \ldots, a_{6}\right)^{T}
\end{aligned}
$$

is an isomorphism between the Lie algebras $G_{2}$ and $R^{6}$, where the matrix $A=V$ is presented in (58). Therefore, we can rewrite the Lax matrices (57) and (58) as follows:

$$
\begin{aligned}
& U=(0,1, \lambda-u, 0,0, v)^{T}, \\
& V=\left(V_{1}, V_{2}, \ldots, V_{6}\right)^{T},
\end{aligned}
$$

where $V_{i}=\sum_{m=-\infty}^{-1} V_{i m} \lambda^{-m}, i=1,2, \ldots, 6$.

The compatibility condition of (64) under the frame of the Lie algebra $R^{6}$ is equivalent to that of (43) under the Lie algebra $G_{2}$.

According to the scheme for generating Hamiltonian structure of integrable couplings by employing the variational identity, a constant symmetric matrix $F$ should be obtained which satisfies the matrix equation

$$
R(b) F=-F(R(b))^{T}, \quad F^{T}=F .
$$

A direct verification shows that

$$
F=\left(\begin{array}{cccccc}
2 \eta_{1} & 0 & 0 & 2 \eta_{2} & 0 & 0 \\
0 & 0 & \eta_{1} & 0 & 0 & \eta_{2} \\
0 & \eta_{1} & 0 & 0 & \eta_{2} & 0 \\
2 \eta_{2} & 0 & 0 & 2 \eta_{2} & 0 & 0 \\
0 & 0 & \eta_{2} & 0 & 0 & \eta_{2} \\
0 & \eta_{2} & 0 & 0 & \eta_{2} & 0
\end{array}\right)
$$

satisfies (65), where $\eta_{1} \neq \eta_{2}$, constants. Define a linear functional

$$
\{a, b\}=a^{T} F b .
$$

With the help of (64), (66), and (67), we get that

$$
\begin{aligned}
& \left\{V, \frac{\partial U}{\partial u}\right\}=-\eta_{1} V_{2}-\eta_{2} V_{5}, \\
& \left\{V, \frac{\partial U}{\partial v}\right\}=\eta_{2}\left(V_{2}+V_{5}\right), \\
& \left\{V, \frac{\partial U}{\partial \lambda}\right\}=\eta_{1} V_{3}+\eta_{2} V_{6} .
\end{aligned}
$$

Substituting the above consequences into the variational identity reads

$$
\begin{aligned}
& \frac{\delta}{\delta \tilde{u}} \int^{x}\left(\eta_{1} V_{3}+\eta_{2} V_{6}\right) d x \\
& =\lambda^{-\gamma} \frac{\partial}{\partial \lambda} \lambda^{\gamma}\left(\begin{array}{c}
-\eta_{1} V_{2}-\eta_{2} V_{5} \\
\eta_{2}\left(V_{2}+V_{5}\right)
\end{array}\right),
\end{aligned}
$$

where $\delta / \delta \tilde{u}=(\delta / \delta u, \delta / \delta v)^{T}$.

Inserting $V_{j}=\sum_{m=-\infty}^{-1} V_{j m} \lambda^{-m}, j=2,3,5,6$, into (69) gives

$$
\begin{aligned}
\frac{\delta}{\delta \tilde{u}} \int^{x} & \left(\eta_{1} \sum_{m=-\infty}^{-1} V_{3 m} \lambda^{-m}+\eta_{2} \sum_{m=-\infty}^{-1} V_{6 m} \lambda^{-m}\right) d x \\
= & (-m+\gamma)\left(\begin{array}{c}
-\sum_{m=-\infty}^{-1}\left(\eta_{1} V_{2 m}+\eta_{2} V_{5 m}\right) \lambda^{-m-1} \\
\eta_{2} \sum_{m=-\infty}^{-1}\left(V_{2 m}+V_{5 m}\right) \lambda^{-m-1}
\end{array}\right) \\
= & (1+\gamma-m)\left(\begin{array}{c}
-\sum_{m=-\infty}^{-1}\left(\eta_{1} V_{2, m-1}+\eta_{2} V_{5, m-1}\right) \lambda^{-m} \\
\sum_{m=-\infty}^{-1}\left(V_{2, m-1}+V_{5, m-1}\right) \lambda^{-m}
\end{array}\right) \\
& +(1+\gamma)\left(\begin{array}{c}
-\eta_{1} V_{2,-1}-\eta_{2} V_{5,-1} \\
\eta_{2}\left(V_{2,-1}+V_{5,-1}\right)
\end{array}\right), \quad m=-1,-2, \ldots
\end{aligned}
$$

If taking $V_{2,-1}+V_{5,-1} \neq 0$, we have $\gamma=-1$. Hence, we get from (70) that

$$
\left(\begin{array}{c}
-\eta_{1} V_{2, m-1}-\eta_{2} V_{5, m-1} \\
\eta_{2}\left(V_{2, m-1}+V_{5, m-1}\right)
\end{array}\right)=\frac{\delta H_{m}}{\delta \tilde{u}},
$$

where $H_{m}=-\int^{x}\left(\left(\eta_{1} V_{3 m}+\eta_{2} V_{6 m}\right) / m\right) d x$ are the conserved densities of the negative-order integrable coupling (51). 
Therefore, (51) can be written as the Hamiltonian structure form

$$
\begin{aligned}
\tilde{u}_{t_{n-1}} & =\left(\begin{array}{c}
2 \partial_{x}\left(V_{2,1-n}\right) \\
-2 \partial_{x}\left(V_{5,1-n}\right)
\end{array}\right) \\
& =\left(\begin{array}{cc}
\frac{2 \partial}{\eta_{2}-\eta_{1}} & \frac{2 \partial}{\eta_{2}-\eta_{1}} \\
\frac{2 \partial}{\eta_{2}-\eta_{1}} & \frac{2 \eta_{2} \partial}{\eta_{2}\left(\eta_{2}-\eta_{1}\right)}
\end{array}\right)\left(\begin{array}{c}
-\eta_{1} V_{2,1-n}-\eta_{2} V_{5,1-n} \\
\eta_{2}\left(V_{2,1-n}+V_{5,1-n}\right)
\end{array}\right) \\
& =J\left(\begin{array}{cc}
-\eta_{1} V_{2,1-n}-\eta_{2} V_{5,1-n} \\
\eta_{2}\left(V_{2,1-n}+V_{5,1-n}\right)
\end{array}\right) \\
& =J \frac{\delta H_{1-n}}{\delta \widetilde{u}}, \quad n=1,2, \ldots,
\end{aligned}
$$

where $J$ is a Hamiltonian operator.

Remark 2. Two negative-order integrable couplings of the negative-order KdV equation were obtained. One of them is linear; the other is nonlinear. We can only generate the Hamiltonian structure of the nonlinear integrable coupling (51) by employing the variational identity; however, up to now, we have not found a suitable method to deduce the Hamiltonian structure of the linear negative-order integrable coupling (38). May its Hamiltonian structure not exist? which is worth of further discussing in forthcoming days. Besides, we observe that the recurrence relations in negativeorder integrable couplings are all nonlocal. In addition, [27] presented some higher dimensional Lie algebras and were devoted to discussion of 2 + 1-dimensional integrable systems, which may be used to produce more interesting negativeorder integrable hierarchies.

\section{Conflict of Interests}

The authors declare that there is no conflict of interests regarding the publication of this paper.

\section{Acknowledgments}

This work was supported by the National Natural Science Foundation of China (Grant no. 11371361), the Natural Science Foundation of Shandong Province (Grant no. ZR2013AL016), and the Fundamental Research Funds for the Central Universities (2013XK03).

\section{References}

[1] B. Fuchssteiner, "Coupling of completely integrable systems: the perturbation bundle," in Applications of Analytic and Geometric Methods to Nonlinear Differential Equations, P. A. Clarkson, Ed., pp. 125-138, Kluwer Academic Publishers, Dordrecht, The Netherlands, 1993.

[2] W.-X. Ma, "Integrable couplings of soliton equations by perturbation I. A general theory and application to the KdV equation," Methods and Applications of Analysis, vol. 7, no. 1, pp. 21-56, 2000 .
[3] Y. F. Zhang and H. Q. Zhang, "A direct method for integrable couplings of TD hierarchy," Journal of Mathematical Physics, vol. 43, no. 1, pp. 466-472, 2002.

[4] F. Guo and Y. Zhang, "The quadratic-form identity for constructing the Hamiltonian structure of integrable systems," Journal of Physics A: Mathematical and General, vol. 38, no. 40, pp. 8537-8548, 2005.

[5] W.-X. Ma and M. Chen, "Hamiltonian and quasi-Hamiltonian structures associated with semi-direct sums of Lie algebras," Journal of Physics A: Mathematical and General, vol. 39, no. 34, pp. 10787-10801, 2006.

[6] W. X. Ma and B. Fuchssteiner, "The bi-Hamiltonian structure of the perturbation equations of the KdV hierarchy," Physics Letters A, vol. 213, no. 1-2, pp. 49-55, 1996.

[7] W.-X. Ma, X.-X. Xu, and Y. Zhang, "Semi-direct sums of Lie algebras and continuous integrable couplings," Physics Letters A, vol. 351, no. 3, pp. 125-130, 2006.

[8] W.-X. Ma, X.-X. Xu, and Y. Zhang, "Semidirect sums of Lie algebras and discrete integrable couplings," Journal of Mathematical Physics, vol. 47, no. 5, Article ID 053501, 16 pages, 2006.

[9] W.-X. Ma, "Variational identities and applications to Hamiltonian structures of soliton equations," Nonlinear Analysis: Theory, Methods \& Applications, vol. 71, no. 12, pp. e1716-e1726, 2009.

[10] Y. Zhang and H. Tam, "A few integrable systems and spatial spectral transformations," Communications in Nonlinear Science and Numerical Simulation, vol. 14, no. 11, pp. 3770-3783, 2009.

[11] Y.-F. Zhang and Y. C. Hon, "Some evolution hierarchies derived from self-dual Yang-Mills equations," Communications in Theoretical Physics, vol. 56, no. 5, pp. 856-872, 2011.

[12] B. L. Feng and J. Q. Liu, "Two expanding integrable systems and quasi-Hamiltonian function associated with an equation hierarchy," Communications in Nonlinear Science and Numerical Simulation, vol. 16, no. 2, pp. 661-672, 2011.

[13] Z. J. Qiao, "Commutator representations of three isospectral equation hierarchies," Chinese Journal of Contemporary Mathematics, vol. 14, no. 1, pp. 41-49, 1993.

[14] Z. J. Qiao, "A general approach for getting the commutator representations of the hierarchies of nonlinear evolution equations," Physics Letters A, vol. 195, no. 5-6, pp. 319-328, 1994.

[15] Z. Qiao, C. Cao, and W. Strampp, "Category of nonlinear evolution equations, algebraic structure, and $r$-matrix," Journal of Mathematical Physics, vol. 44, no. 2, pp. 701-722, 2003.

[16] Z. J. Qiao, "Generation of the hierarchies of solitons and generalized structure of the commutator representation, preprint 1992," Acta Applied Mathematics Sinica, vol. 18, pp. 287-301, 1995.

[17] Z. J. Qiao and J. B. Li, "Negative-order KdV equation with both solitons and kink wave solutions," Europhysics Letters, vol. 94, no. 5, Article ID 50003, 2011.

[18] Z. J. Qiao and E. G. Fan, "Negative-order Korteweg-de Vries equations," Physical Review E, vol. 86, no. 1, Article ID 016601, 2012.

[19] W.-X. Ma and Z.-N. Zhu, "Constructing nonlinear discrete integrable Hamiltonian couplings," Computers \& Mathematics with Applications, vol. 60, no. 9, pp. 2601-2608, 2010.

[20] Y. F. Zhang and H. W. Tam, “Three kinds of coupling integrable couplings of the Korteweg-de Vries hierarchy of evolution equations," Journal of Mathematical Physics, vol. 51, no. 4, Article ID 043510, 2010. 
[21] Y. F. Zhang and E. G. Fan, "Coupling integrable couplings and bi-Hamiltonian structure associated with the Boiti-PempinelliTu hierarchy," Journal of Mathematical Physics, vol. 51, Article ID 083506, pp. 1-18, 2010.

[22] Y. F. Zhang and H. W. Tam, "Four Lie algebras associated with $R^{6}$ and their applications," Journal of Mathematical Physics, vol. 51, no. 9, Article ID 093514, 30 pages, 2010.

[23] G. Z. Tu, "The trace identity, a powerful tool for constructing the Hamiltonian structure of integrable systems," Journal of Mathematical Physics, vol. 30, no. 2, pp. 330-338, 1989.

[24] W. X. Ma, "A new hierarchy of Liouville integrable generalized Hamiltonian equations and its reduction," Chinese Journal of Contemporary Mathematics, vol. 13, no. 1, pp. 79-89, 1992.

[25] Y.-F. Zhang and J. Liu, "Induced LIE algebras of a sixdimensional matrix LIE algebra," Communications in Theoretical Physics, vol. 50, no. 2, pp. 289-294, 2008.

[26] Y. F. Zhang and J. Q. Mei, "Lie algebras and integrable systems," Communications in Theoretical Physics, vol. 57, no. 6, pp. 10121022, 2012.

[27] B. Feng, Y. Zhang, and H. Dong, "A few integrable couplings of some integrable systems and $(2+1)$-dimensional integrable hierarchies," Abstract and Applied Analysis, vol. 2014, Article ID 932672, 9 pages, 2014. 


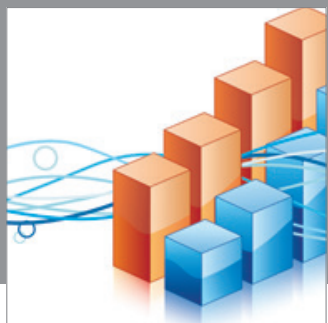

Advances in

Operations Research

mansans

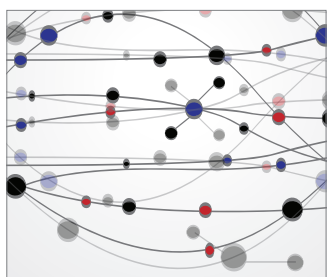

The Scientific World Journal
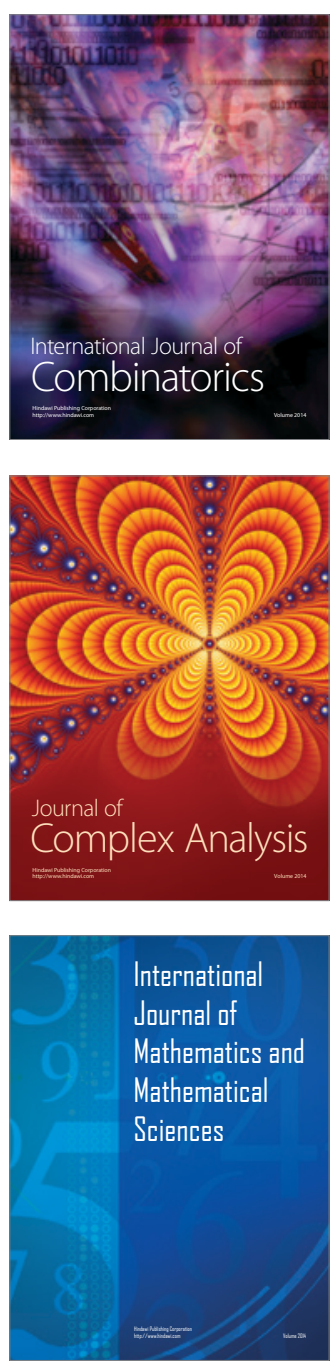
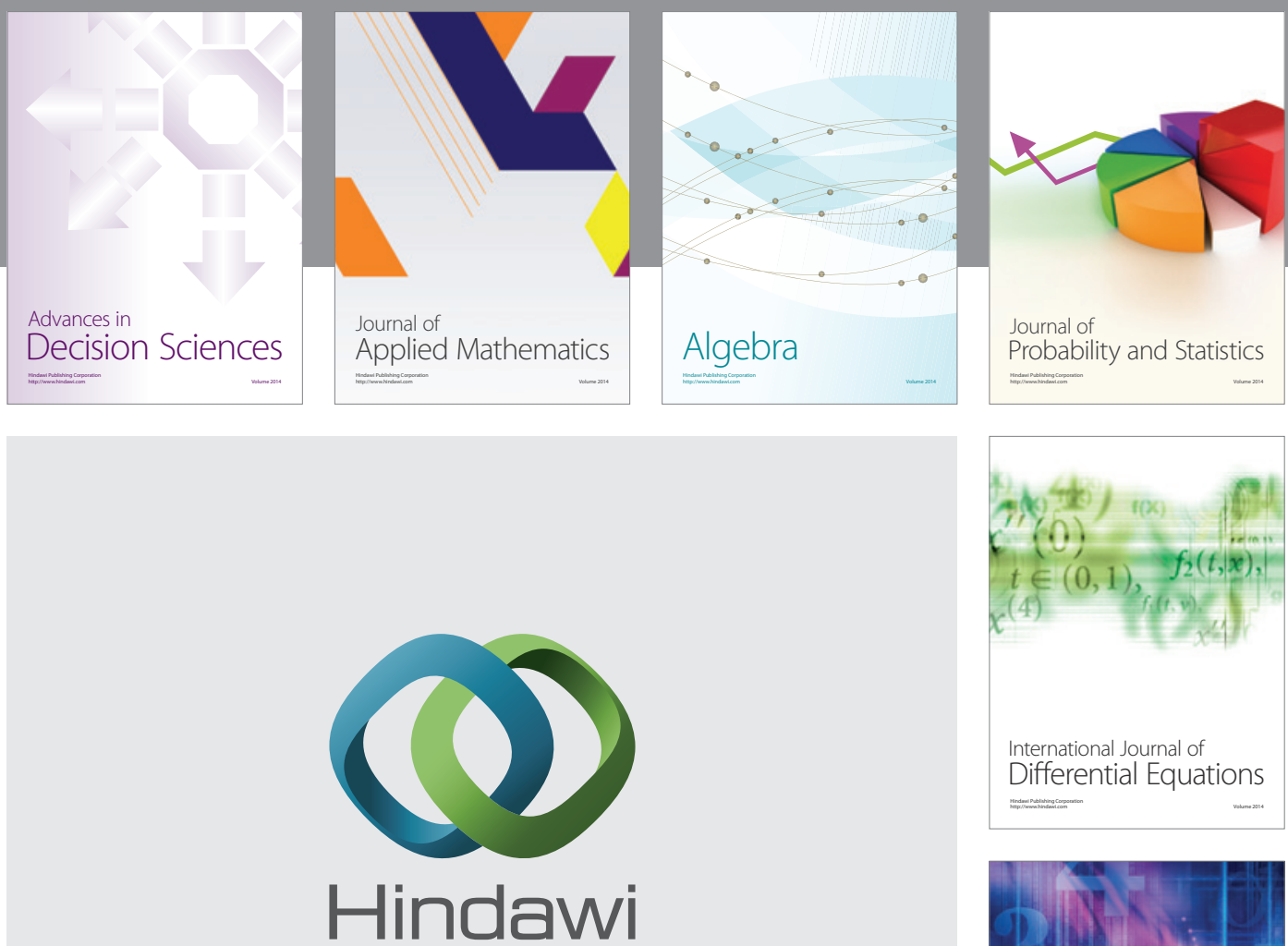

Submit your manuscripts at http://www.hindawi.com
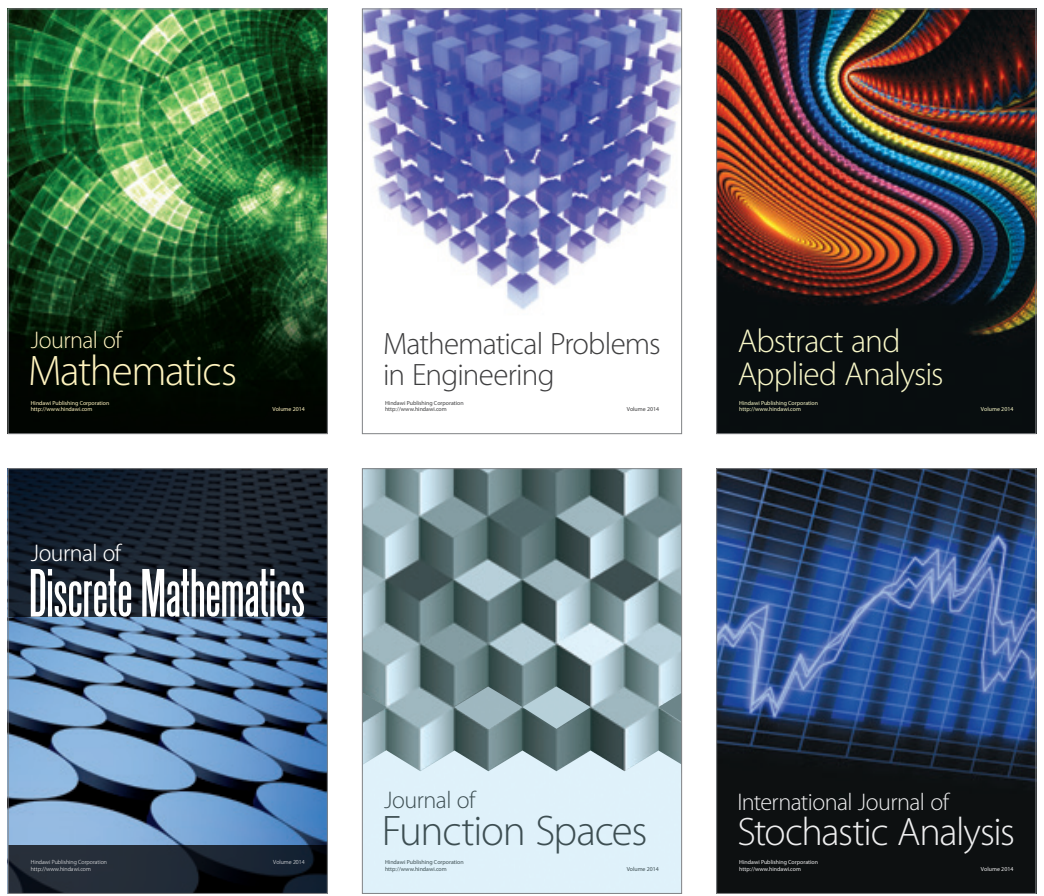

Journal of

Function Spaces

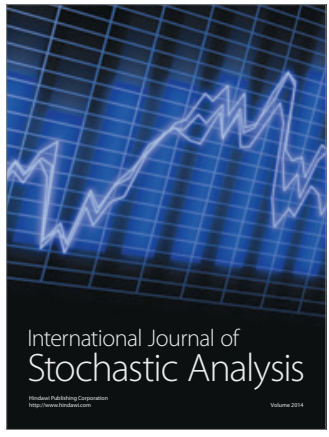

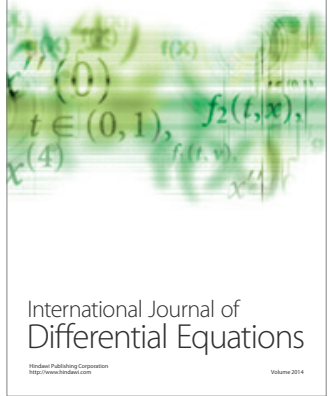
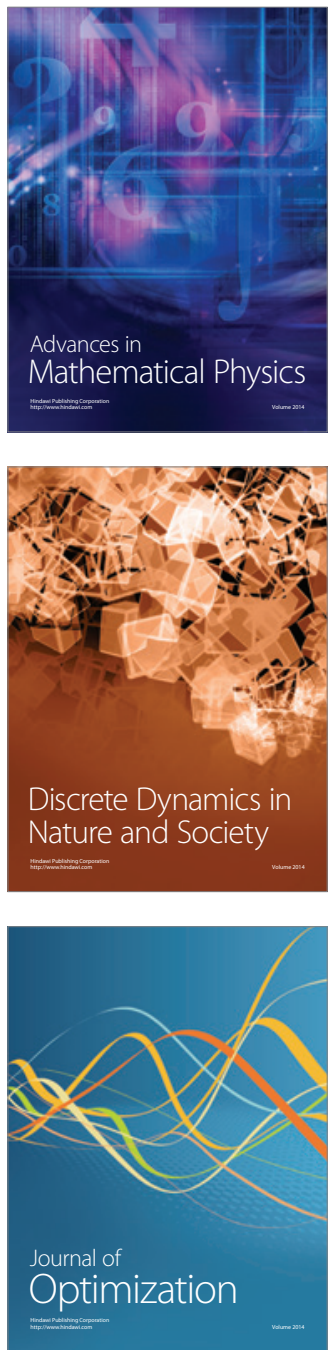